\title{
La democracia directa y la prohibición del mandato vinculante en el debate italiano
}

\section{Direct democracy and the prohibition of the binding mandate: the Italian debate}

\section{RESUMEN}

Uno de los temas más controversiales de los debates políticos en Italia se da acerca de la prohibición del mandato vinculante, consagrado en el artículo 67 de la Constitución italiana. En un contexto de desconfianza colectiva en la política y en la administración de los negocios públicos; un nuevo partido, el movimiento Cinque Stelle, en lo sucesivo M5S, ha alcanzado una suma del $25 \%$ de los votos. Uno de los puntos en el programa de M5S fue la reclamación de sus diputados que son solo voceros de los votantes sin ninguna autoridad sobre estos. Una de las promesas de campaña concernía en presentar proyectos ante los juzgados públicos y parlamentarios a través del uso de una plataforma en línea. Para participar en estos debates los ciudadanos italianos deberían de registrarse en dicha plataforma con una copia escaneada del documento de identidad. Sin embargo, esta plataforma en línea no es neutral, porque pertenece al blog de Beppe Grillo's, quien es el fundador y el líder político del M5S. Sin embargo, él no se movió en la campaña política. Esta circunstancia generó problemas de neutralidad, de privacidad y el uso de los datos personales de los participantes. El personal de Beppe Grillo's ha declarado que la plataforma cuenta con alrededor de 90.000 suscriptores certificados, mientras que los electores del M5S son casi 9 millones. Este es un gran elemento de controversia: ¿cómo podría una minoría participar en el compromiso de los parlamentarios, donde cada diputado representa a toda la nación? Por otra parte, el debate político entre los partidarios del M5S, los críticos y parlamentarios se ha desarrollado en las redes sociales como Facebook y Twitter, sobre todo en las páginas personales de los representantes políticos. Este fenómeno siempre está relacionado con la aparición del Internet en la participación política. El propósito de este artículo es entender cómo se equilibra la contribución de la participación directa del electorado con la prohibición de la unión del mandato parlamentario presente en la Constitución italiana.

\section{PALABRAS CLAVE}

Democracia directa, parlamento, Constitución italiana, electorado, Movimiento Cinco Estrellas. 


\section{ABSTRACT}

One of the most controversial topics of the current political debate in Italy is about the prohibition of the binding mandate previewed by art. 67 of the Italian Constitution. In a context of collective distrust in politics and in the administration of public affairs, a new party, the Movimento Cinque Stelle, (hereinafter, M5S), has exploded collecting $25 \%$ of votes. One of the points of the M5S' program was the claim that its MPs are only spokepersons of the voters, without any autonomy from them. One of the campaign promises was that they would submitted bills to be deposited in Parliament to public judgment through the use of an online platform. To participate in these debates, Italian citizens have to register to the online platform with a scanned copy of their ID document. However, this online platform is not neutral, because it belongs to Beppe Grillo's blog. Beppe Grillo is the founder and the political leader of M5S, but he did not run in the political campaign. This circumstance presents problems of net neutrality, privacy and use of participants' personal data. Beppe Grillo's staff declared that the platform has around 90,000 certified subscribers, whilst the M5S' electors number almost 9 million. This is a huge element of controversy: can a minority be involved in the MPs' commitment, when each MP represents the whole Nation? Furthermore, political debate among M5S' supporters, critics, and MPs has been developed on social network such as Facebook and Twitter, especially on political representatives' personal pages. This phenomenon is connected with the advent of the Internet in political participation and the purpose of this paper is to understand how to balance the contribution of the direct participation of the electorate with the prohibition of binding parliamentary mandate present in the Italian Constitution.

\section{KEYWORDS}

Direct democracy, parliament, Italian Constitution electorate, Movimento Cinque Stelle.

\section{INTRODUCCIÓN}

El Movimiento 5 de Stella (Movimiento Cinco Estrellas, en adelante del M5S), es nueva entidad en el escenario político italiano, que tiene raíces más profundas en el auge de las elecciones políticas del 2013. De hecho en las elecciones política realizadas en febrero 2013, el M5S logró el 25,55\% de los votos, 163 diputados del Parlamento (109 diputados y 54 senadores) convirtiéndose en el partido más votado en Italia (Genga, 2013). Todo comenzó en 2005, cuando Beppe Grillo, un famoso comediante publicó en su blog, convirtiéndose rápidamente en «una de las redes más utilizadas en el mundo en los últimos años» (Brasseur, 2014). Ahora, el blog de Beppe Grillo se ha convertido en un lugar virtual donde difunde sus opiniones políticas, comentadas por cientos de personas (Muller, 2008). De hecho, es sede de distintas actividades, como docenas de «reuniones» ${ }^{1}$, de grupos de activistas que comparten el mismo punto de vista sobre cuestiones políticas, ambientales y sociales. Es decir, la estructura de soporte de la participación política del M5S, son los blogs de grupos políticos locales que participan en los municipios 0 en las administraciones regionales y lo relacionado con las propias actividades Parlamentarias.

A través de su blog, Beppe Grillo organizó tres grandes protestas públicas, el «V-D día» en $2007^{2}$ en Bolonia, $2008^{3}$ en Turín y $2013^{4}$ en Génova. La «V» significa «victoria, venganza, $y$, sobre todo «Vete a la mierda» ( $F^{* *} k$ off») (Muller, 2008). A pesar de utilizar

\footnotetext{
1 Meetup (TM) es una red social, cuyo uso fue sugerido por las actividades de los políticos locales.

2 Grillo también aprovechó la reunión para instar a los italianos a firmar una petición en favor de la introducción de un «Proyecto de Ley de Iniciativa Popular» contra parlamentarios italianos con condenas penales para destituirlos de su cargo.

3 Grillo utiliza la manifestación en contra de «la prensa italiana» y el apoyo financiero que recibe del gobierno; además criticó duramente a la prensa italiana por la falta de libertad.

4 Grillo utiliza para anunciar la participation el rally al 2014. Elecciones al Parlamento Europeo de los M5S.
} 
un insulto bastante vulgar, durante estas reuniones masivas, Beppe Grillo y otros oradores, presentaron a la atención pública el núcleo de su pensamiento político, en especial: la participación directa del público, el servicio público, la protección del medio ambiente, el transporte público, el wi -fi y la ecosostenibilidad.

En la primavera de 2009 en Florencia, se realizó el primer encuentro nacional de "Cinque Stelle» (Bordignon y Ceccarini, 2013), lo que representó la primera experiencia en una elección administrativa. Se trataba de 15 listas cívicas $^{5}$, a raíz de un programa electoral común ${ }^{6}$, en el que las «listas cívicas de Cinque Stelle» obtuvieron 23 representantes municipales. Durante el otoño de 2009 Beppe Grillo habia fundado oficialmente el "Movimento Nacional Cinque Stelle» (M5S) (Bartlett; Frois; Littler; McDonnell, 2013) el cual participó en las elecciones locales y regionales realizadas en el año 2010, ganando con el $3,67 \%$ de los votos dos representantes en el primer lugar y con el $6 \%$ de los votos a dos representantes en Emilia Romaña. En 2011, las votaciones para M5S continuaron en crecimiento, lo que se vio reflejado en las elecciones municipales: en Turín M5S ganó 5,27\% con 2 representantes, Novara $7,77 \%$ con 1 representante, Milán, con 3,45\% con 1 representante, Bolonia 9,41 con 2 representantes. Finalmente en 2012 los M5S ganaron 4 alcaldes. El más importante de ellos fue el de Parma una ciudad cultural e industrial muy conocida en el valle del Po (Povoledo, 2012).

Este movimiento, tuvo un aumento electoral fuerte, especialmente durante las elecciones del 2013, donde por una parte se fundamentó en difundir el descontento popular entre los políticos acusados de corrupción, y por otra parte en sus propios elementos distintivos, los cuales son realmente diferentes a los de otros partidos políticos de gran importancia. En particular, la posibilidad para ser candidato de M5S, se basa en que no debe estar inscrito en otros partidos y no debe tener condenas penales o juicios penales en su contra en otros partidos. Pero el elemento más importante es la promoción de la participación directa en la vida democrática a través de una fuerte crítica a la prohibición de mandato.

\section{LA PROHIBICIÓN CONSTITUCIONAL DEL MANDATO IMPERATIVO}

Los partidos y sindicatos se basan en el concepto de representación. Sin embargo, la representación histórica política no siempre se ha expresado de la misma manera. De hecho, la experiencia de los primeros parlamentos muestra que la regla era la unión del mandato, es decir, la autorización por escrito se daba a los que fueron llamados a representar a dichas clases, comunidades o territorios (Azzariti, 2009), en una perspectiva feudal (Sajó, 1999), en los Estados-Generales de Francia. Básicamente, la actividad del representante se limitaba a una exposición oral de los contenidos de los cuadernos de quejas preparados por el distrito electoral que los eligió (Levico, 2009). Durante la Revolución Francesa el mandato vinculante era un tema a discutir. Tres días después de que el Juramento del Juego de la Corte de Luis $\mathrm{XVI}$ trató de gestionar los eventos que causaron su caída, se promulgó la ordenanza en junio de 1789 donde se declara la prohibición del mandato político vinculante. Este acto abrió el camino hacia el fin del sistema feudal de la representación política que caracteriza el Antiguo Régimen (Azzariti, 2009).

Posteriormente, la prohibición del mandato imperativo se convirtió en el núcleo de la vida democrática moderna, ya que accedieron a definirse en términos concretos de equilibrio entre los poderes y las autoridades políticas. Sin embargo, la responsabilidad del agente, de aclarar un hecho fundamental, no estaba regulada. Durante la redacción de la Constitución de 1793, Robes Pierre puso en duda la responsabilidad de los representantes y funcionarios públicos hacia el "pueblo soberano», con su

5 Se relaciona con las listas cívicas de Treviso, Florencia, Prato, Bolonia, Italia, Roma, Turín, Pescara, Perugia, Bergamo, Nettuno, Spoleto, Forlì, Pozzuoli y Fiumicino.

6 Fue escrito en la «Carta di Firenze» (Carta de Florencia). 
famoso discurso «Sobre la constitución y el gobierno representativo» del 10 de mayo 1793.

Los jacobinos de inmediato se dieron cuenta de que una vez que la autonomía política de los representantes se había garantizado, la rendición de cuentas del mandato era deficiente y por lo tanto la esencia política de la conexión entre el representante y el representado debía ser mejorada. Siguiendo las teorías de Rousseau, y de Robespierre argumentando que la soberanía popular no puede ser representada y que el dominio de la gente sólo dura un día «y, en cualquier caso, «sus delegados son corruptibles».

Esta toma de conciencia hace que la caída de la ilusión de la voluntad de los ausentes (los representados) pueda encontrar su expresión inmediata en los que están presentes (el representante). Esto nos lleva al necesario debate sobre la forma de mantener la comunicación entre los votantes y los funcionarios electos. Incluso doscientos años más tarde, estas críticas no han perdido su relevancia, y como, los métodos de participación y control de los representantes de los gobernados deben todavía ser proporcionados en muchos sistemas jurídicos ${ }^{7}$. En Italia, la prohibición del mandato imperativo se afirma por el art. 67 de la Constitución italiana ${ }^{8}$. Afirma que «Cada miembro del Parlamento representa a la Nación y ejerce sus funciones sin un mandato vinculante». Según algunos doctrinantes de la prohibición del mandato imperativo (Azzariti, 2010), lo que lleva a una libertad de acción de los diputados después de su elección, muestra un componente ideológico; que se trasladó a un lado del candidato, donde los votantes pueden unirse o no a través de su voto en las elecciones. Sin embargo, si el elemento ideológico se desplazó a los votantes, no debe haber una situación similar a la del Antiguo Régimen: donde los votantes deben elegir un programa específico con un referendo y luego elegir los responsables de lo que suceda en el Parlamento. Así, los votantes tienen que seguirlos día a día en el cumplimiento de sus tareas y eventualmente sustituir a los representantes de acuerdo a las normas de derecho privado si no cumplen, pero esto es precisamente lo que el artículo 67 de la Constitución italiana pretende prohibir.

El principal problema de la violación está relacionado con graves escándalos de la incapacidad para gobernar, la corrupción de los miembros del Parlamento que ocurrió en 2008 y 2010 (Ainis, 2010). En el primer punto hay un juicio penal pendiente ante el Tribunal de Nápoles. Los escándalos se vieron agravados por el hecho de que la ley electoral no prevé la elección directa de los candidatos por los electores, pero sólo en la lista ya formada por la secretaría de los partidos. Esta ley ha sido declarada inconstitucional por la sentencia $N^{0} 1 / 2014$ de la Corte Constitucional por violación del derecho de los ciudadanos a elegir a sus propios representantes políticos (Dickmann, 2009).

Actualmente, las respuestas son muy variadas, por ejemplo, la superación de los partidos tradicionales como organismos burocráticos y autorreferenciales, ya no participan en la defensa efectiva de los intereses de sus electores o de los ciudadanos. Este tipo de política representativa podría ser sustituida por más organizaciones, tales como las nuevas formas de movimientos políticos (Algostino, 2007), o con un mayor uso de la democracia directa, a pesar de las difíciles experiencias del referendo en Italia ${ }^{9}$, 0

7 La prohibición del mandato imperativo nunca ha sido formalizado plenamente en los países de tradición anglosajona, aunque se ha impuesto la vida política. De hecho, en las elecciones presidenciales de los Estados Unidos para el mandato vinculante se prevea en los Colegios Electorales (A. Levico, arriba, n. 21, p. 245).

8 En una perspectiva comparada, la prohibición del mandato imperativo. También se afirma en el artículo 27 de la Constitución francesa de 1958, el artículo 38 de la Ley Fundamental alemana de 1949, el artículo 67 de la Constitución Española de 1979.

9 En Italia el referéndum más importante fue en 1946 entre monarquía y república, nuestra forma actual de gobierno. Después de décadas de silencio en las consultas públicas, el referéndum fue principalmente utilizado por el «Partido Radical» (Partido Radical) como herramienta política para reivindicar los derechos civiles. El punto de inflexión fue en 1974 cuando grupos católicos proponen un referéndum para derogar la ley de divorcio, pero fracasó. De hecho, el Partido Radical ha de referendos propuesta contra la ley oscurantista desde 1981. Desde el año 2000, cuatro se deroga referendos no alcanzó el quórum dice el artículo No. 75 de la Constitución italiana. Sólo en 2011, el quórum que se superó widly En cuanto al referéndum sobre la energía nuclear utiliza, así como que en la privatización de los servicios públicos esenciales. 
la democracia participativa (Allegretti, 2008). Donde la propuesta política de M5S encajaría en este escenario perfectamente.

\section{PROPUESTAS M5S EN LAS OPCIONES POLÍ- TICAS Y LA DEMOCRACIA DIRECTA}

La propuesta de M5S sobre cuestiones de representaciones políticas es un híbrido entre la democracia directa y la democracia participativa con el objetivo fundamental de la superación de las prohibiciones del mandato imperativo.

La democracia directa está diseñada para permitir a los ciudadanos interesados en los proyectos de legislación y enmiendas constitucionales para la aprobación de los votantes (en el poder de la iniciativa) y pedir que las leyes sean aprobadas por los organismos legislativos referidos por los votantes «para su aprobación o rechazo» (el poder de referendo popular). El ejercicio de la democracia directa es común sobre todo en los Estados Unidos ${ }^{10}$ y Suiza $^{11}$. A veces es difícil equilibrar la democracia directa con los derechos humanos fundamentales. La pregunta es: ¿si un derecho es inviolable, no podría ser sometido a un referendo sobre su existencia, cuyo resultado es la reservada a la mayoría de los votantes?

Por ejemplo este tipo de problemas se pudieron observar durante los debates, sobre la convivencia entre los derechos de la madre a la intimidad y a la autodeterminación y la prohibición de aborto presentado por referendo en muchos Estados de los Estados Unidos (Carter, 2011), o también la conocida "Proposición 8» de la votación sobre la prohibición constitucional del matrimonio del mismo sexo en California (Burnett, M. D. McCubbins, 2009); y la iniciativa del referendo de introducir una prohibición constitucional sobre la construcción de ciertas maneras aprobadas por los votantes suizos en 2009 (Langer, 2010). De lo contrario, el consentimiento de democracia participativa de los ciudadanos interesados en participar de las decisiones públicas en los que se permiten. Este tipo de participación sobre diversos grados de presencia de los ciudadanos de las instituciones públicas, la mayoría de las cuales muestran la subordinación de esta presencia en los mecanismos de los poderes políticos y administrativos. Por ejemplo, entre muchos ejemplos de democracia participativa no son los presupuestos participativos con experiencia en algunas Ciudades de América del Sur como Porto Alegre, sino algunos tipos de participación pública en el debate público en asuntos urbanos o ambientales (Allegretti, 2011).

La democracia participativa de la M5S es dirigida de dos formas por un lado a través del sistema operativo, el debate en línea y la aplicación consultante sobre el blog de Beppe Grillo sobre el borrador de proyectos de ley en el Parlamento representativo de la M5S; y por otro lado se maneja la participación directa del grupo de trabajo de los ciudadanos en problemas específicos tratados por el M5S en grupos de concejales y miembros del parlamento municipal y regional, donde los participantes deberán inscribirse a sí mismos en este blog que les da el número y una copia escaneada de su documento de identidad. Entonces, tienen que ser «certificados» por el personal del blog. En consecuencia, los participantes registrados pueden participar tanto en la votación como en los procesos de discusiones.

\footnotetext{
10 En los Estados Unidos, la democracia directa tiene sus fundamentos en la filosofía de Thomas Jefferson, aunque nunca abogó por este tipo de prácticas. En concreto, según él para la «confianza en la sabiduría y la bondad de la mayoría numérica» combinado con «una profunda desconfianza del gobierno» dado lugar a un gran respeto por la voluntad del pueblo.

11 En Suiza, se utilizó el modelo de referéndum desde el final de la Edad Media. Sin embargo, fue suprimida en los siglos XVII y XVIII, en consecuencia la formación de las oligarquías locales. Reapareció en el siglo XIX. En ese período la mayoría de los cantones aceptaron el encargo de presentar sus constituciones y leyes para el pueblo. En 1848, imitando a los Estados Unidos, Suiza se convirtió en una federación, y la nueva constitución fue puesto a la gente para su ratificación en la gran mayoría de los cantones. Más tarde, cada uno la enmienda a la Constitución Federal tiene que ser aprobada por referéndum obligatorio Aunque las boletas se ampliaron en 1874, de manera opcional, para cubrir cualquier ley federal aprobada por el Parlamento. Iniciativas populares, lo que conduce a un referéndum obligatorio, originalmente se les permitió sólo para revisión total de la Constitución federal.
} 
El rol de Beppe Grillo en el blog de actividades políticas de M5S fue fundamental ya que se consideraba su principal sede política. Teniendo en cuenta su función específica de adicionador de las actividades políticas y el punto de conexión entre los parlamentarios, como voceros, y votantes, cómo se organiza. A primera vista, es muy compleja y llena de información. La plantilla del blog está dividida en tres, seleccionadas verticalmente en diferentes tamaños: uno de los principales problemas sobre todo las opiniones del mismo Beppe Grillo, también el alojamiento de diferentes puntos de vista y opiniones críticas sobre las actividades de otros partidos políticos. Junto a ella se encuentra la segunda sección llamada «minipost», en el que el núcleo de la información publicada es el desarrollo de actividades políticas o de los representantes electos en M5S. La tercera columna de la plantilla que refiere a la difusión de los principales artículos de prensa y vídeos de televisión que trata sobre las actividades políticas del partido.

El núcleo de las actividades políticas M5S no está en el centro de la plantilla, pero se trasladó a la parte superior derecha y contiene enlaces en el formulario de inscripción para el sitio y sus instalaciones, tales como las plataformas "reuniones» y el "S $\mathrm{O} »^{12}$. Incluso si el $\mathrm{S} \mathrm{O}$ debe ser el núcleo del debate político M5S puede ser un instrumento eficaz para acceder a la democracia participativa, que presenta un fuerte límite a la participación, ya que es accesible sólo a personas registradas y "verificadas» que realizaron el proceso antes de 30 de junio 2013, la fecha de la última declaración actualizada. Fue una circunstancia que permitió de hecho la formación de una élite, cuya consistencia no estaba clara, ya que no se disponía de datos personales de los participantes registrados al público, por razones obvias de privacidad. Este hecho podría ser evaluado bajo el «código de conducta» ${ }^{13}$, firmado por cada candidato de la M5S antes de 2013 en elecciones políticas e implementado en el estatus del grupo parlamentario. Que se trataba de muchos elementos relacionados con la «relación» entre electores y diputados M5S.

El código de conducta de los diputados del M5S se inspiró en la transparencia de los ciudadanos a través de informes periódicos sobre las decisiones políticas tomadas por la votación en el Parlamento. Los parlamentarios tendrán que mantener una relación con los suscriptores a través de la implementación de sus propuestas durante el período de la legislatura. El objeto principal de los parlamentarios electos es la implementación del Programa de M5S, en particular, con los principios de la democracia directa, como referendos sin quórum, y el debate parlamentario obligatorio con una votación pública sobre los proyectos de ley en que se promueven la participación popular y la elección directa de los miembros de la votación parlamentaria. Donde el Parlamento tiene que estar motivado y explicando a diario con un video publicado en el medio de YouTube M5S.

La afirmación de que M5S dejaría solo a los diputados o voceros de los votantes, sin ningún tipo de autonomía frente a ellos, al establecer su código de conducta que:

Las solicitudes de proyectos de ley que surgen desde el portal M5S por los usuarios registrados deben ser llevadas a la sesión parlamentaria si se votan al menos un $20 \%$ de los votantes registrados. Los grupos parlamentarios todavía tendrían que ser capaces de evaluar cada propuesta, incluso si está por debajo del umbral del $20 \%$.

Sin embargo, la consulta de los miembros verifica que la plataforma ya había sido hecha a través del blog, incluso antes de la implementación del sistema operativo, que se produjo el 28 de octubre de 2013. Estas consultas estaban ya listas, por

12 También hay enlaces a las versiones en inglés y japonés, ambos con contenidos simplificados en comparación a la versión italiana.

13 Código de Conducta electo Movimiento 5 Estrellas en el Parlamento. 
ejemplo: la elección del nombre y la posición en la lista electoral de cada candidato de la M5S en las elecciones nacionales de 2013 (conocida como «Parlamento», fue una palabra inspirada por una adaptación específica del modelo americano de las "elecciones primarias»), regionales (conoce como Regional) y las elecciones municipales; como las elecciones de las candidaturas de oficinas públicas para posiciones tales como el Presidente del Senado y el Presidente de la República Italiana (conocido como «Quirinarie»), la opción política de si la inmigración ilegal tiene que ser tratada como delito o no, las opciones de los requisitos fundamentales de la nueva ley electoral es discutida por el Parlamento; la decisión de reunirse con el primer ministro designado, la expulsión de seis parlamentarios, diputados y senadores, del grupo parlamentario M5S. Es una circunstancia que se declaró explícitamente en el código de conducta:

La expulsión del grupo parlamentario de la M5S. Los diputados M5S y parlamentarios tienen que cumplir, sin distinción entre los miembros de la Cámara y el Senado. En caso de violaciones claras del Código de Conducta, la expulsión de un diputado tiene que ser propuesto por la mayoría de los diputados de la M5S. La expulsión debe ser ratificada por el voto en línea en el portal M5S entre todos los participantes, por la mayoría de votos.

Este procedimiento presenta problemas relevantes. Cómo el personal de Beppe Grillo declara que la plataforma cuenta con alrededor de 90.000 suscriptores certificados, mientras que el número de electores del M5S fue de casi 9 millones. Este es un gran elemento de controversia: porque ¿puede una minoría participar en el compromiso de los parlamentos, cuando cada diputado representa a toda la nación?

\section{LA DEMOCRACIA DIRECTA, LA PROPORCIO- NALIDAD Y LA RENDICIÓN DE CUENTAS A LOS VOTANTES}

La democracia participativa puede ser útil para el intercambio de conocimientos, habilidades y experiencias, pero la solución propuesta para la puesta en práctica de la democracia directa a través de un blog de propiedad privada podría tener algunos problemas que podrían ser interesantes de investigar.

Primeramente, una pregunta tiene que ser contestada: por ejemplo ¿Quiénes son estas personas? (Aroney, 2006). Más de 9 millones de votantes eligieron al M5S en las elecciones 2013, y ¿cuántos de ellos están involucrados en la votación a través del blog? La última experiencia, la expulsión de 4 senadores del grupo de parlamentario de la M5S, vio sólo unas decenas de miles de suscriptores registrados que participaron de la votación en línea. De acuerdo con la declaración de Beppe Grillo difundida por sí mismo a través de Twitter, 43.368 suscriptores certificados participaron en la votación en línea: 29883 a favor de la expulsión y 13.485 contrario a ella. ¿Son una muestra relevante que puede limitar los senadores y diputados a la voluntad de la mayoría de ellos? ¿Qué tipo de problemas se presentan? Matemáticamente, se representan el $0,003 \%$ de los votos obtenidos por M5S en las elecciones de febrero de 2013. No parece ser un voto de confianza, porque carece de la representatividad de los votantes del M5S, ya que no persigue el principio de la proporcionalidad entre el fin perseguido y (la responsabilidad de los representantes frente al representado) y la herramienta utilizada para lograrlo, sobre todo el sistema de votación en línea (Sweet y Mathews, 2008).

Por otra parte, las razones por las que se fundamentan el voto en línea en su expulsión parecían estar más vinculadas a las opiniones personales sobre las estrategias políticas del M5S que a una asignación específica de delitos (Mumelter, 2014). La falta de debate entre los representantes y los suscriptores de la plataforma en línea, y el voto en línea en los propios representantes sobre su estancia en el grupo parlamentario, infringieron la dignidad del Parlamento y de los propios diputados de acuerdo con el artículo 67 de la Constitución italiana (Ruggeri, 2011).

El representante se sienta en las bancas de la Cámara o el Senado y representa a toda la nación, y no sólo a la pequeña parte de los votantes que 
participaron en la votación en línea. Si no que además, la certificación de cada abonado, así como el registro de acceso a la plataforma de votación en línea, no garantiza el anonimato, y así la libertad del voto. Además, los gerentes y los propietarios de la plataforma siempre sabrán el manejo del voto. Donde califican los suscriptores con datos estrechamente vinculados a las personales, debido a la inscripción de los documentos de identidad de los votantes. En el cual la circunstancia permite que el perfil de cada usuario se de en función de su voto y de opinión, y la limitación de su libertad en cuanto al derecho a ser olvidado: ¿cuánto tiempo están estos datos relacionados con los votos y opiniones expresadas guardados por los administradores y propietarios del sistema de votación en línea? Nadie sabe a ciencia cierta (D'Elia, 2012). De hecho, si este tipo de voto aparentemente puede dar una sensación de gratificación a los votantes exasperados por «la impunidad de los políticos». Sin embargo, este sistema evita la discusión y el debate, por lo tanto, es probable que se convierta en un referendo sobre el personal del diputado, independientemente de las razones por las que él o ella eligieron disentir del grupo o las reglas de los mismos.

El enfoque propuesto por M5S olvida que la prohibición del mandato imperativo pasa a los políticos electos una tarea que no refleja la realidad representada por ellos mismos, como el modelo M5S le gustaría llevar a cabo. En cambio, los políticos tienen que realizar una función de representación, «en el sentido de que deben representar una cierta idea de la sociedad, no simplemente sus votantes» (Zagrebelsky, 2009:76). Así, el modelo propuesto por M5S podría arriesgarse para legitimar a sus votantes de base, así como a los grupos de presión económica y tecnocrática, como una nueva forma de feudalismo, fragmentando los intereses generales.

¿Cómo es posible reclamar la responsabilidad de los representantes frente a sus electores sin quitarle su independencia gratuita? Un análisis comparativo de este problema podría sugerir una solución diseñada bajo el principio de proporcionalidad: en el proceso de recuperación. Como sugiere su nombre, la «recuperación» es una manera de quitar a un funcionario electo antes de finalizar del plazo normal del desarrollo en un trabajo (Feeney, 2007). Incluso si está escasamente investigada por estudiosos, al proceso de recuperación que tiene una tradición de constitucionalidad fructífera para demostrar la historia estadunidense demostrando ser un importante dispositivo de vigilancia que debe estar a disposición de las personas, que tiene una conexión estricta con la soberanía popular y el modelo republicano (Zick,1999). Sin embargo, una monarquía constitucional de larga tradición como la británica ha sido el desarrollo de su introducción desde la crisis de los gastos durante el último Parlamento. De hecho, se ha tomado como modelo en 26 sistemas legales en todo el mundo, así como en los Estados Unidos, Argentina, Canadá (Columbia Británica), Filipinas, Corea del Sur, Suiza y Taiwán. Donde los constituyentes podrían reclamar la rendición de cuentas de los diputados en casos específicos establecidos por la ley, como la delincuencia suficientemente grave como para recibir una sentencia, tomando el soborno, haciendo comentarios racistas u ofensivos, alegando los gastos que no les corresponden, el cambio a una política de diferente partido, rompiendo una promesa en su folleto electoral. En efecto, las normas específicas tienen que ser proporcionadas al solicitar la retirada por los mandantes. Estatutos del recuerdo pueden variar de un Estado a otro, sin embargo, siguen siendo un modelo general de tres pasos. En primer lugar, los votantes tienen que circular a una petición para recoger un número suficiente de firmas. En segundo lugar, los funcionarios electorales tienen que determinar si la petición de recolectar los requisitos establecidos por la ley y el número de firmas válidas para que se lleve a cabo. En tercer lugar, si se llevan a cabo los dos elementos de la retirada (Weinstein, 2005).

En Italia no existe la destitución. Sin embargo, el M5S publicó las nuevas reglas para la aplicación de la elección 2014 al Parlamento Europeo y entre estas reglas se propuso una idea muy original de destitución que afirma:

El representante electo debe decir si él o ella son culpables de un delito penal. En el caso de que él 0 ella deban ser acusados de un delito penal, él o ella 
tendrán derecho a decidir si abandonan la oficina 0 no. El diputado también debe decidir si él o ella no violaran el código de conducta con el compromiso de dar cumplimiento de sus normas suscritas en el momento de presentación y de su solicitud respecto a los criterios de la M5S. El diputado será considerado culpable de falta grave, de acuerdo con el principio de democracia directa conocido como «recuperación», como ya se ha aplicado en los Estados Unidos: por lo menos en 500 miembros de la M5S como desde 31/12/2012 residiendo en la jurisdicción en la que el diputado fue elegido de manera unánime. Donde se ha propuesto para declarar una violación grave; si la propuesta ha sido aprobada por una mayoría de votos en la red M5S por los suscriptores de los de la M5S desde el 30/06/2013 residen en la jurisdicción en la que el miembro fue elegido.

Una vez más, se propone exclusivamente a los usuarios registrados en la plataforma antes del 31 de diciembre de 2012, por lo que los electores que realmente eligieron a los diputados europeos estuvieran excluidos, tanto de reclamar la propia memoria como de votar en ella. Sin embargo, la votación en línea se extiende a los suscriptores registrados hasta antes del 30 de junio de 2013. Eso parece significar que no todos se liberaron de tener las mismas posturas y parece que sólo las personas registradas a finales de 2012 tienen un disfrute pleno de los derechos en el voto.

También debe tenerse en cuenta que sólo los 500 suscritos que residen en la circunscripción pueden reclamar el procedimiento de «recuperación». Es un número muy pequeño, en relación con los votantes, que podrían aumentar la incertidumbre de la actividad política del diputado, o someterla a alguna presión indebida.

Una implementación efectiva de este modelo en el sistema italiano puede dar lugar a una mejora en el debate sobre la responsabilidad de los funcionarios, electos y evitar la fragmentación y la confusión del debate sobre responsabilidades típicas de las experiencias en línea del M5S y los políticos a través de la red social, como diputados electos y otros medios como Facebook y Twitter o Beppe Grillo.
Estos funcionarios con «blog propio». Sin embargo, la propuesta M5S está utilizando el término «recuperación», pero sin ella la sustancia y limitándola a su propio sistema.

\section{LOS TEMAS CRÍTICOS SOBRE EL MODELO M5S DE VOTACIÓN EN LÍNEA: EN EL CASO «PARLAMENTARIO»}

El modelo de votación en línea de M5S podría también ser discutido a través del "Parlamento» que tuvo lugar en diciembre de 2012. En ese caso, los suscriptores certificados votaron para elegir los nombres y la posición de los senadores y diputados, los candidatos en las listas electorales de febrero 2013 en las elecciones generales. El número limitado de participantes fue un argumento constante con respecto a los debates sobre M5S. Esta experiencia podría ser muy interesante porque está estrictamente conectada a la visión práctica de la prohibición del mandato imperativo y la realización del proceso de votación en línea colectiva. El Parlamento el cual se puso en marcha a través de la solicitud en línea de Beppe Grillo. Donde la mayoría de las críticas formuladas por la opinión pública y los comentaristas políticos afectaron tanto los problemas y a su neutralidad en la plataforma y el pequeño número de los votantes.

En relación con el primer punto, los observadores dijeron que los resultados de las votaciones finales parecían faltar la parte tercera y la neutral de la certificación. De hecho, desde un punto de vista técnico no había controles sobre la neutralidad de los sistemas operativos, ni de las medidas de seguridad. Sobre el segundo punto, como ya se ha dicho antes, las críticas de voto de los senadores y las expulsiones de preocupación y ponderación dieron como resultado la falta de proporcionalidad; más bien, la elección del nombre y la posición de los candidatos subraya que está completamente cerrado a los extranjeros, convirtiendo el resto del electorado en espectadores pasivos. En el fondo de esta cuestión, hay una contradicción fuerte para resolver. Primeramente se refiere al conflicto entre la participación, y luego al abrir de las más amplias contribuciones posibles que se hacen también 
desde los grupos de afuera de los activistas, ya que se caracterizó a los orígenes de la V-días, y la preservación de la identidad, para evitar una adquisición hostil, lo que justifica la restricción de los participantes en la votación en línea. Donde los números hablan claro: los suscriptores certificados al sitio fueron 255.339 participantes. Sólo 31.612 de ellos se inscribieron y se certificaron el 30 de septiembre 2012. Sólo $12,38 \%$ de los suscriptores tienen derecho a expresar sus preferencias, y entre ellos solamente 20.252 votaron por la composición de la lista, mientras el electorado italiano en general es más o menos por 50.449.979 electores. Se trata de una cuestión ideológica muy fuerte, una paradoja en un partido político que quería abrir las instituciones a los ciudadanos como «latas de atún», porque en este punto, son los que controlan si la gente puede o no ser parte de M5S a través de la certificación que se tiene sobre la misma M5S, que ha tratado de dar una solución a este problema a través de la extensión de la certificación a todos los elementos: la certificación de los candidatos, de los votantes, y a los miembros, y así sucesivamente.

Pero la idea misma de la participación política, es un elemento esencial de la libertad, en comparación con la misma prohibición del mandato imperativo, parece estar orientada a la lógica subyacente de la ley de marcas privadas. De hecho, en este sentido, se trata de los grandes temas sobre la propiedad de Beppe Grillo de la marca M5S utilizado como símbolo político. Seguido por este camino de la interpretación es probable que la privatización de toda la relación entre en el símbolo político de electo M5S y la entidad de referencia Beppe Grillo y el cuerpo electoral, de los problemas extremos relacionados con un mandato imperativo, en una dirección que podría escapar de los mismos promotores y participantes de la M5S.

La democracia directa, las redes sociales y la participación en línea y el debate político entre los partidarios de la M5S, son críticos y parlamentarios que se han desarrollado en las redes sociales como Facebook y Twitter, en especial en las páginas personales de representantes políticos que provocan su extrema fragmentación. Esta circunstancia se vie- ne alentando por el uso de la red social masiva por activistas de la M5S. Por ejemplo, es obligatorio para aquellos que quieran participar en los procesos de selección de candidatos, hacer una presentación en video de sí mismos, para gestionar su propia página de Facebook y así sucesivamente. Sin embargo, como un fuerte apoyo al uso de las redes sociales hace que el efecto sea paradójico de hacer una división entre las personas cuya opinión puedan afectar la acción política, ya que la certificación antes de una fecha determinada puede darse en la plataforma, y los que deben limitarse a expresar su papel como pasivos espectadores a través de los comentarios. Es bien sabido que las redes sociales tanto el O. S. son de propiedad privada, por lo que varios grupos de la M5S activistas a nivel local sugieren la conversión de sus experiencias de acuerdo con Wikipedia.

Liquid Feedback (abreviado) es un software libre para la formación de la opinión política y toma de decisiones, que combina aspectos de la democracia representativa y directa. Su característica más importante es la implementación de un sistema de voto delegado («Democracia Líquida»), que es el que está encargado de establecer una nueva forma de representación política y la participación que tiene en cuenta la disparidad de conocimientos de sus participantes.

Sin embargo, la solución LQFB no fue aceptada por M5S, que prefiere usar su aplicación específica haciendo hincapié en su estrategia de propiedad. Este tipo de elección parecería confirmar la propensión de la M5S de «democracia de audiencia», según lo descrito por Manin (1997) y destacando el uso de Internet. En este modelo, los partidos políticos reconocen un amplio espacio y oportunidades para la personalización y la comunicación que de cualquier manera han podido hacerse cargo de la organización de los partidos tradicionales. Las consecuencias son que las entidades colectivas, por una parte, son como los partidos, que son los recolectores tradicionales de participación, que se debilitaron. Por otro lado las relaciones entre los partidos políticos, la sociedad civil y los votantes pasan casi exclusivamente a 
través de los medios de comunicación y márquetin político (Gallo, 2013). A menudo, el debate público en internet no parece favorecer la mediación entre los diferentes puntos de vista políticos y los intereses contrastantes. De hecho, la creación de grupos de internet conectados en los lazos de parentescos entre el "amigo» y la hostilidad contra los «enemigos comunes» es más fuerte que en los circuitos políticos tradicionales. Que reduce las posibilidades de encontrar puntos de encuentro entre los lados opuestos donde podrían encontrarse grandes fracturas que aún existen en las comunidades. Estas circunstancias favorecieron la tendencia a «tomar partido» basadas en eslóganes publicitarios en lugar de establecer un diálogo ponderado. A través del análisis de cómo el debate político tiene lugar fuera del Parlamento es lo esencial que es la «igualdad de representación de cada voto», siendo cada voto libre e independiente de su fuente electoral político (Zanon, 2014).

\section{EL COMPROMISO FORMAL DE LOS CANDI- DATOS Y FUNCIONARIOS ELECTOS AL PARLAMENTO EUROPEO}

Entre las varias nuevas reglas que los candidatos y representantes electos en el Parlamento Europeo deben firmar para ser inscritos en las listas electorales de la M5S, no están particularmente criticadas sobre el incumplimiento del código de conducta, en violación de la prohibición del mandato imperativo.

Antes de las elecciones, cada candidato de la M5S del Parlamento Europeo debe firmar un compromiso formal de cumplir con el código de conducta, emprendiendo específicamente el compromiso de renunciar como diputado en caso de condena y en los casos en que se considera que están en grave incumplimiento del código de conducta y respeto. Si se encuentra en incumplimiento él o ella debe pagar la cantidad de $€ 250.000$ a la Comisión Europea de Organización Electoral y la M5S la donará a la caridad.

Este tipo de compromiso no es aplicable debido a la prohibición bien conocida del mandato otorgado por el artículo 67 de la Constitución italiana vinculante y es nula de pleno derecho de acuerdo con la legislación de la UE. De hecho, el considerando $\mathrm{N}^{\circ} 4$ del artículo No. 2 y el artículo $N^{\circ} 3$ de la Decisión del Parlamento Europeo de 28 de septiembre 2005 tomaron la adopción del Estatuto de los diputados al Parlamento Europeo (2005/684/CE,). En concreto, el artículo 2 establece que «1. Los diputados serán libres e independientes. 2. Los acuerdos relativos a la renuncia del mandato de un Miembro antes 0 al final de una legislatura, será nula y sin efecto»; y el artículo 3 afirma que: «1. Los miembros votarán de manera individual y personal. No estarán sujetos a instrucciones ni recibirán mandato imperativo alguno. 2. Los acuerdos relativos a la forma en que el mandato se ha de ejercer será nulo».

Por otra parte, en esta cláusula no es válido el código de conducta de la M5S donde hay dos elementos que permiten confirmar el carácter privado de la relación con la política fundada por la M5S y sus representantes. Ellos son la firma de la cláusula de penalización de $€ 250.000$ y la especificación de la intención de donar los fondos pueden ser recopilados para una organización benéfica sin especificar. Incluso si son legalmente exigibles y de acuerdo a un mero punto de vista político, tanto de estos estados que tienen un valor simbólico y representan el precio de la violación de la prohibición del mandato imperativo y de la libertad de elección de la MP, negando la esencia de la libertad del mandato y el establecimiento de una posible servidumbre política. De hecho, aunque esta regla no es aplicable en frente de los tribunales o el Parlamento de la UE, que tiene una persuasión muy fuerte para los suscriptores de la M5S, por lo que la sanción real de los diputados en el M5S podría ser perder la rol en la comunidad. Esto es suficiente para limitar la libertad.

\section{CONCLUSIONES}

Los estudiosos sugieren que la propuesta fundamental de la M5S de la prohibición del mandato imperativo hace un renacimiento de los «días de los pioneros de su nacimiento en la Europa continental durante 
ese taller de Derecho Constitucional establecido en Francia entre 1789 y 1793. Sin embargo, el modelo M5S va más allá de la crítica conceptual del artículo 67 de la Constitución Italiana, ya que propone cambiar el modelo del ejercicio de soberanía popular. En efecto, el artículo 1, y 2 de la Constitución italiana afirman que "La soberanía reside en el pueblo y se ejerce por el pueblo en las formas dentro de los límites de la Constitución», pero la idea de la M5S es mover este ejercicio a la «web», específicamente a su idea de Internet, que la identifica con un medio certificado para la M5S.

Los suscriptores, para los cuales los funcionarios electos son voceros. Son a aquellos a los que se refieren estrictamente a la tradición jacobina, asumiendo también el atributo de «ciudadanos» para indicar a los diputados, el concepto de «representación» que es casi inadmisible. De hecho, el enfoque de la M5S rechaza la idea misma de «representación», que significa "dar presencia a los ausentes», y la noción de la toma de decisiones en nombre y por cuenta de las personas representadas, como el resultado de la evaluación independiente del representante. El revolucionario «sans-culottes» participo en las sesiones de la Convención de la Revolución francesa donde se sustituyen de forma moderna por las sesiones plenarias, a través de la «gente de la web» y ejercer un control constante, que con una «línea se haga clic en «aprobar y apoyar o contradecir y deslegitimar. De hecho, la democracia digital, en lugar de la democracia representativa, debe exigir la abolición de la prohibición del mandato imperativo como la» piedra angular «del sistema constitucional italiano. Por otra parte, la teoría del M5S en la política del mandato es ciertamente compatible con la del uso de la Internet, pero es «sin duda incompatibles» con el modelo parlamentario representativo.

El levantamiento de la prohibición del mandato imperativo intencionalmente podría resolver el problema de la corrupción y el comportamiento irresponsable de los políticos. Sin embargo, está relacionada con una aceptación social y cultural por el conjunto del propio electorado y no puede ser resuelto por la cancelación de una garantía constitucional relevante. Los políticos a los que los votantes dependen para su representación, están llamados a representar a una sociedad en la que los votantes creen que, porque la planificación del tiempo presente y futuro es el objetivo de los funcionarios políticos electos existe un alto riesgo de transformación de estos medios en medios extremos. En otras palabras, mediante el uso de instrumentos tecnológicos para alcanzar ciertos objetivos, los políticos tienden a imponer sus propias decisiones a la ciudadanía, mientras que la adopción de una técnica aparentemente se tornaría neutral.

\section{REFERENCIAS BIBLIOGRÁFICAS}

- Genga N. (2013). Movimiento 5 Estrellas, partido líder y ganador moral, en el federalismo, 05 de marzo 2013, http://www.federalismi.it/Appl OpenFilePDF.cfm?artid=21978\&dpath=documen t\&dfile=05032013102637.pdf\&content=MoVimento+ $5+$ Stelle:+primo+partito+e+vincitore+morale++stato+-+dottrina+-+

- Brasseur. (2014). Internet y la política: el impacto de las nuevas tecnologías de la información y la comunicación en la democracia, Consejo de Europa, Asamblea Parlamentaria, Informe | Doc 13.386 | 13 enero de 2014.

- Muller T. (2008). El infierno de Beppe, The New Yorker.

- Bordignon F., Ceccarini L.(2013). Cinco Estrellas y Cricket. Beppe Grillo sacude la política italiana, Sur Sociedad Europea y la Política, Volumen 18, número 4. pp. 427-449.

- Bartlett J., Froio C., Littler M., McDonnell D. (2013). Nuevos actores políticos en Europa: Beppe Grillo y los M5S, Londres. Demos.

- Povoledo E. (2012). Mapa Político del Comediante cáustica Altera Italia, New York Times, 18 de Mayo de 2012. http://www.nytimes.com/2012/ 05/19/world/europe/caustic-comedian-altersitalys-political-map.html?action=click\&module= Search\&region=searchResults\%230\&version= \&url=http $\% 3 \mathrm{~A} \% 2 \mathrm{~F} \% 2 \mathrm{Fquery}$. nytimes.com $\% 2$ Fsearch $\% 2 F$ sitesearch $\% 2 F \% 3$ Faction $\% 3$ Dclick\%26contentCollection\%3DN.Y.\%2520\% 252F\%2520Region\%26region\%3 DTopBar\% 
26module\%3DSearchSubmit\% 26pgtype $\% 3$ Darticle\%23\%2FBeppe\%2520Grillo

- Azzariti G. (1990). Los ciudadanos, partidos políticos y grupos parlamentarios: todavía hay una prohibición de carácter obligatorio, la Democracia y la Ley de 2009. N. Zanon, el derecho constitucional de mandato parlamentario libre, Giappichelli,

- A. Sajó (1999). Gobierno Limitación: Una introducción a Constitucionalismo, Central European University Press, Budapest.

- Levico A. (2009). Tasa X. La historia de un signo, phoenix, Boves.

- Incorvati G. (2010). Rousseau, el príncipe y los principios de los derechos políticos modernos, en G. Incorvati, F. Marcelli, Crisis de la democracia y la crisis de los partidos políticos en Italia y en todo el mundo, Aracne, Roma.

- Azzariti G. (2010). La crisis de los partidos como una crisis de su capacidad representativa, en G. Incorvati, F. Marcelli, Crisis de la democracia y la crisis de los partidos políticos en Italia y en todo el mundo, Aracne, Roma.

- Ainis, M. Corrupto y corruptor, La Stampa, 12/ 12/2010,http://www.lastampa.it/2010/12/12/cultura/opinioni/editoriali/corrotti-e-corruttoriHcY5GA1EGlaCUkE2mt5xSM/pagina.html; M. Guerzoni Cambio parte, el fiscal abrió una investigación, Corriere della Sera, 11/12/ 2010,http://archiviostorico.corriere.it/2010/ dicembre/11/Cambi_partito_Procura_ap32 Corte Cost. 13.1.2014 No. 1.

- Dickmann R. (2009). La Corte declara inconstitucional la mayor parte de la prima y las listas de votación y se introduce un sistema de representación proporcional basado en una preferencia pura. www.federalismi.it.

- Greblo E. (2011). Filosofía de Beppe Grillo. Movimiento 5 estrellas, Milán - Udine.

- Algostino A. (2007). La democracia y sus formas. Una reflexión sobre el movimiento No Tav, en la Ley de Política.

- Allegretti U. (2008). Democracia Participativa y los procesos de democratización, la Democracia y Derecho.
- Carter E. (2011). Aborto reguladora un Través de la democracia directa: la libertad de Todos Versus El Código Moral de la Mayoría, y 91 BUL Rev. 305.

- Burnett CM, McCubbins MD, (2012). Sexo en urna: percepción de iniciativas de ley respecto del mismo sexo contraer matrimonio y el aborto en California. http://dx.doi.org/10.2139/ssrn. 1884579; GB Lewis Gossett GW, ¿por qué pasar californianos Proposición 8?, 2009 http:// ssrn.com/ABSTRACT $=1451709$

- Langer L. (2010). Panacea o Falacia Patética? La prohibición de los minaretes en Suiza, $43 \mathrm{~V}$ y. J. Transnat'l L. 863, p. 867; D. Moeckli, de minaretes y criminales extranjeros: suizos democracia directa y de los Derechos Humanos, Derechos Humanos Law Review, 2011, p. 774.

- Allegretti U. (2011). La democracia participativa, en Italia y en Europa, la Asociación Italiana de constitucionalistas Magazine, No. 1.

- Aroney N. (2006). Formación, Representacion y Modificacion baño federales las Constituciones, 54 Am. J. Comp. L. p. 277.

- Sweet AS, Mathews J. (2008). Proporcionalidad Equilibrio y Global Constitucionalismo, 47 Colum. J. Transnat'I L. 72, p. 116.

- Mumelter G. (2014). Psicodrama, internacional Cinco Estrellas.http://www.internazionale.it/ opinioni/gerhard-mumelter/2014/02/27/ psicodramma-a-cinque-stelle/.

- Ruggeri A. (2011). Notas para un estudio sobre la dignidad humana, de acuerdo con el derecho constitucional, Revista de la Asociación de Constitucionalistas.

- D'Elia D. (2012). Voto en línea Movimento 5 Stelle: insegura y poco transparente, La Republica. http://www.tomshw.it/cont/news/votoonline-movimento-5-stelle-insicuro-e-poco-trasparente/ 41677/1.html

- Zagrebelsky G. (2009). L'inganno. Experiencia y política técnica, en R. Lissoni (ed), Prometeo y la democracia, Albo Versorio Edizioni, Milano.

- Feeney F. (2007). Recordemos el 2003 California gubernativa, 41 Creighton L. Rev. 37.

- Zick T. (2004). El consentimiento de los gobernados: Retiro del Mercado de senadores de 
Estados Unidos, 103 Dick. L. Rev. 567, (1999), p. 571. Véase una visión crítica en J. OsborneKlein, Electoral Recall en el estado de Washington y California: Necesidades California Normas más estrictas para proteger a los funcionarios electos de Acoso, 28 Seattle Univ. L. R. 145.

- Cámara de los Comunes- Comité Político y de Reforma Constitucional. (2012). Retiro del Mercado de los Diputados, Primer Informe de la Sesión 2012 un 13, Londres.

- Manin B. (1997). Los principios del gobierno representativo, Cambridge - Nueva York.
- Gallo F. (2013). Democracia 2.0. La Constitución, los ciudadanos y la participación, http://www. cortecostituzionale.it/documenti/convegni_ seminari/20130915_Gallo.pdf, p. 3.

- Zanon N. (2014). La segunda técnica juventud. 67 de la Constitución. http://www.forumcostitu zionale.it/site/images/stories/pdf/documenti_ forum/giurisprudenza/2014/0007_nota_1_ 2014_zanon.pdf.

- Weinstein R. le encienden!, (2005)Versión los votantes de «The Apprentice»: un análisis de Recall elecciones locales en California, $15 \mathrm{~S}$. Cal. Interdis. L. J. 131. 